\title{
Measurements and Visualization of Sound Intensity Around the Human Head in Free Field Using Acoustic Vector Sensor
}

\author{
JÓZEF KOTUS ${ }^{1}$ AND BOŻENA KOSTEK, ${ }^{2}$ AES Fellow \\ (joseph@sound.eti.pg.gda.pl) \\ (bokostek@audiacoustics.org) \\ ${ }^{I}$ Multimedia Systems Department, Faculty of Electronics, Telecommunications and Informatics, Gdansk University of \\ Technology, Gdansk, Poland \\ ${ }^{2}$ Audio Acoustics Laboratory, Faculty of Electronics, Telecommunications and Informatics, Gdansk University of \\ Technology, Gdansk, Poland
}

\begin{abstract}
This paper presents measurements and visualization of sound intensity around a human head simulator in a free field. A Cartesian robot, applied for precise positioning of the acoustic vector sensor, was used to measure sound intensity. Measurements were performed in a free field using a head and torso simulator and a setup consisting of four different loudspeaker configurations. The acoustic vector sensor was positioned around the head with a 5-cm step. Sound intensity was measured in 277 points. For every step three orthogonal sound intensity components were calculated. Pure tones of frequencies 250,1000 , and $4000 \mathrm{~Hz}$ were applied to analyze the acoustic field. Obtained results were used to provide visualizations of sound intensity distribution around the human head. The tool developed for this purpose utilized three-dimensional sound intensity measurements and visualization techniques.
\end{abstract}

\section{INTRODUCTION}

The main goal of this paper is to measure and visualize the real flow of the acoustic vector field around the human head in several configurations using a sound intensity measurement technique. The authors' main intention was to examine and visualize the complexity of the acoustic energy flow around the human head in a stereo-based loudspeaker configuration [1] in a well-controlled condition, i.e., free field. This was performed in the context of an assumption that if two loudspeakers produce equally loud sounds, then signals at both ears are identical; thus-in theorythere are no apparent time-of-arrival differences and the sound image is perceived as a "phantom center" image, and it should be equal to the one received from the center speaker. This phenomenon has been very thoroughly measured and reported in literature, but the previous research has been based on sound pressure measurements. However, as the last years have seen the introduction of anemometric transducers that are capable of directly measuring the acoustic particle velocity field [2][3], some major changes to measuring and characterizing sound field by means of intensity metric quantities rather than only by scalar quantity, i.e., sound pressure, were proposed. Typically, intensimetric quantities are used for measuring energy transmission and propagation paths, as well as for detection of noise source localization [4], determination of acoustic impedance, and reflection index of materials [2][5], although one may find several examples of employing them in audio engineering [6][7] and in particular in recording and reconstruction of the acoustic field, e.g., ambisonics [8]. An interesting application of sound intensity technique for analysis and visualization of directional room responses was presented in 2001 during the 111th AES Convention in the USA [9]. The authors applied the 3-D microphone array for intensity calculation (p-p intensity probe) for directional analysis of the measured room responses. Since impulse responses are inherently multidimensional, they introduced a new way of visualizing 5-dimensional data and showed its usefulness in demonstrating time-frequency properties of the responses [9].

In relation to sound field analysis, substantial literature sources contain publications in which the results of analytical model calculations are verified by experimental tests. Such an analysis concerns mainly a distribution of pressure levels, i.e., a scalar parameter of an acoustic field. However, in a real acoustic field, there is a close relationship between scalar and vector effects, represented by the acoustic pressure and particle velocity. The scalar-vector description of acoustic field characteristics can be represented by 
both potential and kinetic energy. They both account for physical interpretation of wave phenomena and render the mechanisms of propagation, radiation, diffraction or scattering [5][10]. A valuable illustration of the scalar-vector phenomena occurring in real conditions is the application of the sound intensity technique in tests where the product of the acoustic wave pressure and particle velocity is measured by means of an appropriate measurement probe $[2][3][11][12]$. There are a lot of examples that provide visualization of acoustical wave phenomena around obstacles in different configurations. Most of them concern the interaction of acoustic waves with obstacles such as sphere, cone, screen, and others. Unfortunately, measurement results of the acoustic field distribution around the human head with the use of the vector field (some preliminary measurements results were presented by the authors during 136th AES Convention in Berlin [13]) are not really existent. On the other hand, multiple simulation results for these issues can be found in literature [14][15]. Also, we can find papers that explore WFS (Wave Field Synthesis) virtual sound sources that are perceived to be at locations between loudspeakers and the listener and the amount of perceptual artifacts that occur in the synthesized field [16]. It should be noted that utilizing methods based on sound intensity rather than pressure may also be found in the literature in a different context. For example Ando and Hamasaki proposed a new 3-D panning method based on the coincidence of sound intensities at the receiving point. They demonstrated that the method introduced yields the same results as the one based on sound pressure besides the total weighting applied for the loudspeaker arrangement [17].

It is important to emphasize that in general, analytic models provide useful information about pressure acoustics, pressure fields, but none currently offers a full vector mapping of the acoustic energy flow in front of and behind obstacles. Interference, diffraction, and scattering of waves make the real field very complex and challenging in terms of creating truly faithful theoretical frameworks. Taking these facts into consideration, the authors decided to perform measurements of the vector acoustic field around the human head using the sound intensity technique.

The acoustic energy distribution was obtained using a sound intensity measurement technique. The 3-D Acoustic Vector Sensor (AVS) [2][3][10] was applied for this purpose. Details of the applied measurement procedure were recorded along with the description of the calibration procedure. Phase mismatch may strongly affect the accuracy of sound intensity measurements performed in the source near field, thus a proper calibration of the AVS requires that velocity and pressure transducers are closely matched in phase and compensated for nonlinearities of the spectral characteristics. Presentation of pathways through which the acoustic energy is conveyed is especially useful in visualization of acoustic sources and in explaining their effects in real-life conditions [12]. In this paper the main focus was put on the accuracy of the sound field measurements and the resulted analysis and visualization.

\section{EXPERIMENTAL SETUP}

\subsection{Equipment Applied for Sound Intensity Measurements}

The list of equipment and software included USP (Ultimate Sound Probe) Probe with the USP Conditioning module; Cartesian Robot; B\&K Pulse System, type: 7540; B\&K Time Data Recorder software version: 17.0; B\&K Head and Torso simulator, type: 4128C-002; 4-channel amplifier; SLA4 and 4 REVEAL 601p speakers; sound intensity analysis software prepared by the authors; software created for visualization working in the MATLAB environment. Selected devices are described below in more detail.

\subsection{Acoustic Vector Sensor}

In recent years, it is often observed that sound field measurements employ both anemometric transducers and a pressure microphone that allow simultaneous and coincident measurement of sound pressure and acoustic velocity vector. Therefore, a single acoustic vector sensor measures acoustic particle velocity directly instead of measuring sound pressure, which is measured by conventional microphones. The AVS measures air velocity across two tiny resistive strips of platinum that are heated up to approximately $200^{\circ} \mathrm{C}$ to provide temperature difference. The sensor itself is very small: typical dimensions of the heated wire are $5 \mu \mathrm{m}$ in diameter and 1 to $3 \mathrm{~mm}$ in length, thus giving a nearly pin-point measurement. It operates in a flow range of $10 \mathrm{~nm} / \mathrm{s}$ up to about $1 \mathrm{~m} / \mathrm{s}$. Each particle velocity sensor is sensitive in only one direction, therefore, three orthogonally placed particle velocity transducers are used. In combination with a pressure microphone, the sound field in a single point is fully characterized, and the acoustic intensity vector, which is the product of pressure and particle velocity, can be determined. This intensity vector indicates the acoustic energy flow. With a compact probe, the full three-dimensional sound intensity vector can be determined within the audible frequency range from $20 \mathrm{~Hz}$ up to $20 \mathrm{kHz}$ [2][3].

The Microflown sensor does not require a membrane and an associated mechanical vibration system. It is also free from various forms of mechanical system response limitations. Unlike pressure-gradient microphones, velocity probes do not measure acoustic pressure at two points to derive a pressure gradient. When particle velocity is present, acoustical particle velocity sensors measure temperature difference of two closely spaced and heated platinum wire resistors and quantify particle velocity from the temperature measurement [7].

It is evident that it is much more complicated to calibrate the Microflown sound intensity probe than a p-p sound intensity probe. On the other hand, the Microflown probe is much smaller than the p-p intensity probe, which is an advantage in many applications, especially in the measurements described [18].

However, irrespective of the measurement principle used in measuring the particle velocity, there is one fundamental problem: the pressure and the particle velocity transducer will invariably have different phase responses. One must 


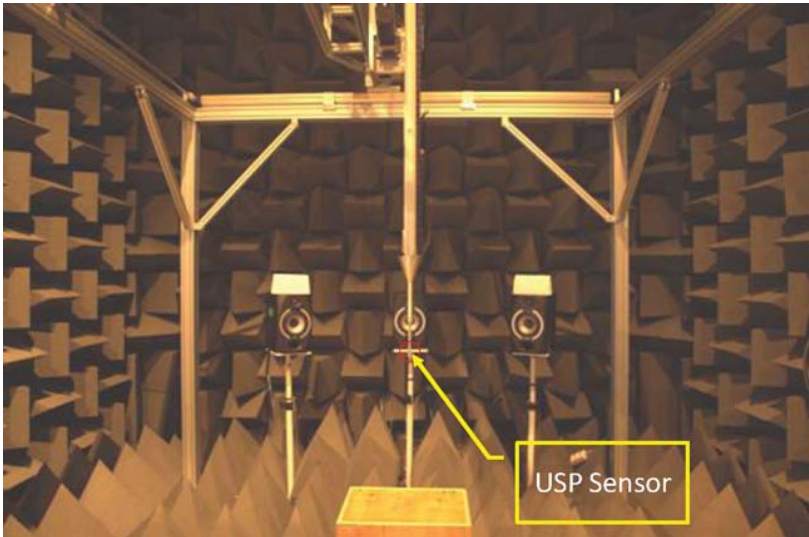

Fig. 1. Cartesian robot installed in an anechoic chamber. The USP sensor placed in the reference position; calibration in progress.

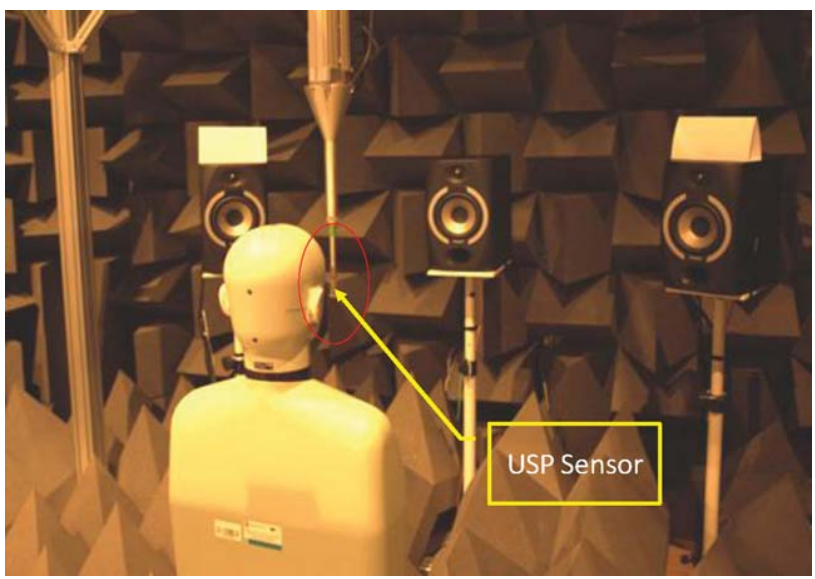

Fig. 2. Cartesian Robot installed in an anechoic chamber along with a head and torso simulator; measurements in progress.

compensate for this "p-u phase mismatch," otherwise the result may well be meaningless. In fact, even a small residual $\mathrm{p}$-u mismatch error can have serious consequences under certain conditions [19]. The phase correction methodology applied for this purpose is described in detail in Sec. 2.4.

\subsection{Cartesian Robot}

For precise positioning of the acoustic probe, a specialized Cartesian robot was designed and practically implemented (Figs. 1 and 2). Its design guarantees fixed space orientation of the acoustic probe. The robot can place the measurement probe with a positioning accuracy of 200 $[\mu \mathrm{m}]$. Ranges of motion are: $\mathrm{X}-1850, \mathrm{Y}-2000$, and $Z-1540[\mathrm{~mm}]$. The movement speed for a particular axis is as follows: $\mathrm{X}-50, \mathrm{Y}-50, \mathrm{Z}-15[\mathrm{~mm} / \mathrm{s}]$. For the measurement in question, the applied area was equal to: 800 $\times 800[\mathrm{~mm}]$. The acoustic vector sensor was positioned around the dummy head with a $5-\mathrm{cm}$ step. Sound intensity was measured in 277 points for the constant height ( $\mathrm{Z}$ axis). The grid of measurement points is depicted in Fig. 3.

Positions of loudspeakers and reference points are also shown. More detailed information about the loudspeaker's position and configuration applied during performed measurements is given in Sec. 2.3.

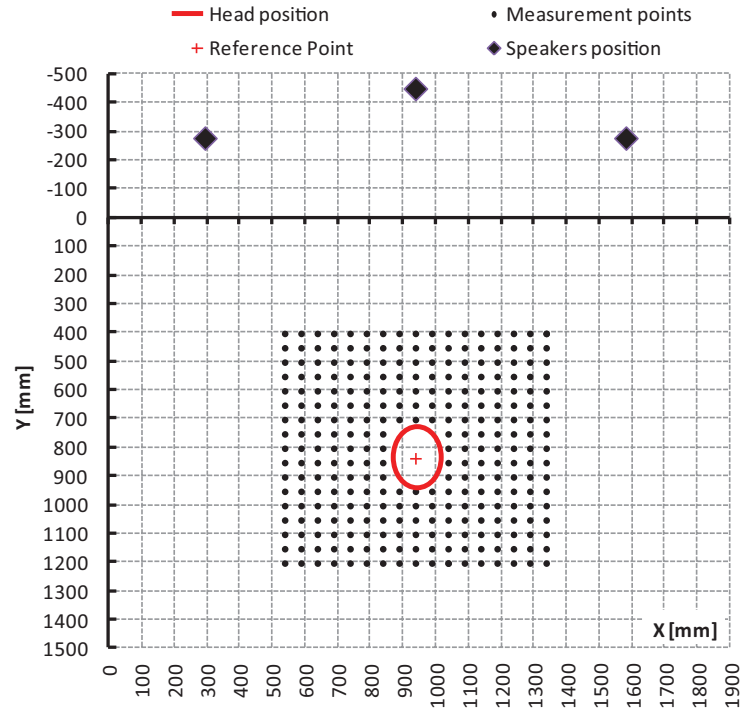

Fig. 3. Grid of measurement points in relation to the head position.

\section{METHODOLOGY OF MEASUREMENTS OF SOUND INTENSITY AROUND THE HUMAN HEAD}

The detailed information about methodology of the performed measurements is presented in this section.

\subsection{Measurement Conditions}

The examination of the energy distribution around the human head was performed in the anechoic chamber. The head and torso simulator was placed $0.6[\mathrm{~m}]$ above the floor and situated on a hard wood table. The height of the measurement surface was $1.2[\mathrm{~m}]$. This height corresponded to the location of the ear canals in the artificial ears mounted in the dummy head. The setup of the speakers, distance to the artificial head, and the sensor have been selected to provide a free-field measurement.

\subsection{Measurement Procedure}

Sound intensity measurements were carried out in two steps. First, the measurement and synchronization signals were recorded according to the procedure presented in Fig. 4.

After loading coordinates of the measuring points into the robot control software, the measurements were started. In the movement phase the sensor position is checked. If the sensor position is correct, linear drive motors switch off to avoid unwanted noise and vibrations that could disturb the measurements. Otherwise, the positioning procedure is performed again. During the sound intensity measurement, the additional synchronization signal was generated in a separate channel. It was recorded using PULSE system simultaneously with measurement signals. The synchronization signal was used during the offline analysis. It enabled unambiguous assignment of every measurement result to the position in which it was captured. After finishing the current measurement, the linear drive motors were switched on again and the measurement sensor was moved to the 


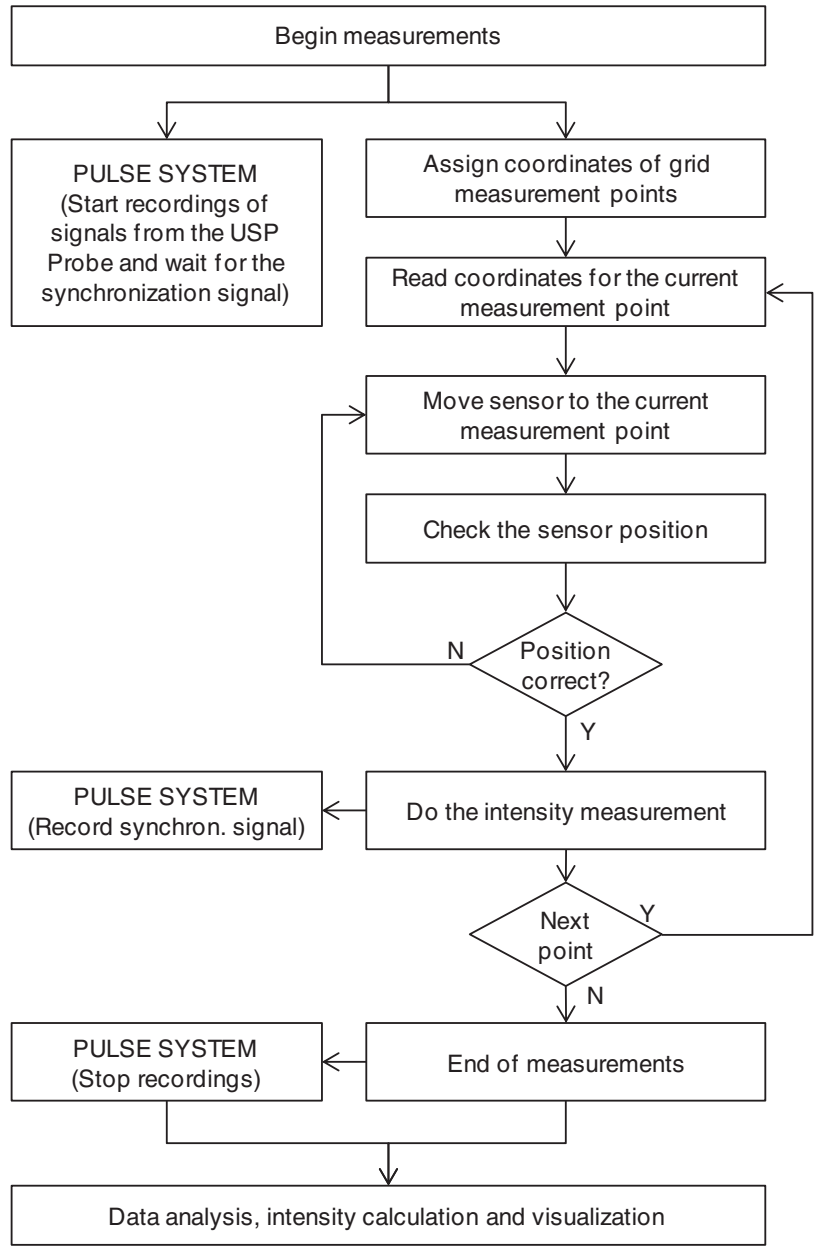

Fig. 4 Procedure of the sound intensity measurements.

next position. The procedure of positioning and measuring was finished when the measurements were performed in all positions.

In the next step the recorded signals were used for offline calculations of sound intensity values. The detailed information about applied signals and the method of sound intensity calculation is presented in the following sections. The starting point was to introduce the grid of measurement point coordinates into the application that was tasked with controlling the Cartesian robot movement. Next, simultaneous recording of measurement and synchronization signals was started. When the robot moved the sensor to the measurement point its drives were switched off and the synchronization signal was recorded in a separate channel. This helped to avoid disturbance of the sound intensity signals. Additional control procedures guaranteed the correctness of the sensor positioning during the whole measurement.

\subsection{Measurement Procedure}

During the measurement of the intensity field distribution around the human head, the multi-harmonic signal was used. Pure tones of frequencies 250,1000, and $4000 \mathrm{~Hz}$ were utilized. All signals had the same amplitude. Test signals were presented and measured in two separate sessions. During the first session, sound intensity was measured in the prepared grid of measurement points without the head and torso simulator. The second session included the head and torso simulator with sound intensity positioned in the same grid of points. During both measurement sessions, test signals were simultaneously played back through the speakers in four configurations:

- First configuration: only the front left loudspeaker was used;

- Second configuration: the front right loudspeaker was used;

- Third configuration: center loudspeaker was used;

- Fourth configuration: the front left and right loudspeakers were used (in-phase).

Loudspeakers were placed according to the AES recommendation [1]. The physical relationship between the head simulator and the two speakers is fixed; they are positioned at the corners of an equilateral triangle. Distance from the front of a given loudspeaker to the reference point was equal to 1.288 [m] (see Fig. 3). Before the measurements, the sound pressure level was checked in the reference point for all loudspeaker configurations separately and set to the same value. It means that every loudspeaker radiated the same acoustic power during the whole measurement session.

\subsection{Sound Intensity Calculation}

Sound intensity is the average rate at which sound energy is transmitted through the unit area perpendicular to the specified direction at the considered point. The intensity in a certain direction is the product of sound pressure (scalar) $p(\mathrm{t})$ and the particle velocity (vector) component in that direction $u(\mathrm{t})$. The time-averaged intensity $I$ in a single direction is given by Eq. (1) [11]:

$$
I=\frac{1}{T} \int_{T} p(t) u(t) d t
$$

Using the presented AVS, the particular sound intensity components can simply be obtained based on Eq. (1). The sound intensity vector in three dimensions is composed of the acoustic intensities in three orthogonal directions $(x, y$, $z$ ), and is given by Eq. (2):

$$
\vec{I}=I_{x} \vec{e}_{x}+I_{y} \vec{e}_{y}+I_{z} \vec{e}_{z}
$$

In the applied algorithm of sound intensity calculation, the time average T (Eq. (1)) was 4096 samples (with the sampling frequency of $48 \mathrm{kHz}$ ). It means that the direction of the sound source was updated more than 10 times per second. A single intensity measurement takes 1 second.

Sound intensity calculation can be performed in the time domain or in the frequency domain [20][21]. Due to the fact that the multi-harmonic signal was employed in the measurements, a method for calculating the sound intensity in the frequency domain was applied for this purpose. Its block diagram is depicted in Fig. 5. The sound intensity calculation algorithm required two types of signals. The first group of signals was delivered by the multichannel acoustic vector 


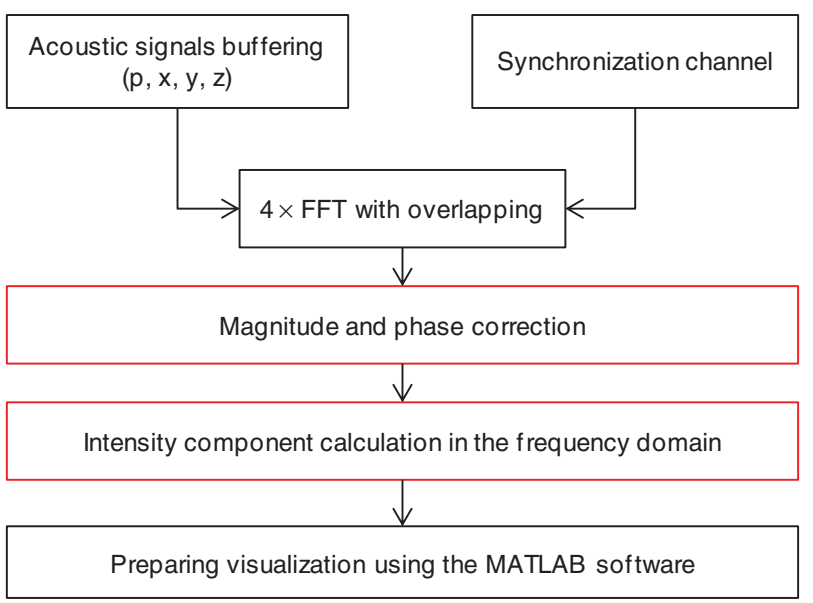

Fig. 5. The block diagram of the algorithm applied for calculating the sound intensity in frequency domain.

sensor. It produced the following signals: sound pressure $p$ and three orthogonal particle velocity components $u_{x}, u_{y}$, $u_{z}$. The second type of signal was applied to synchronize the calculation with the position of the sensor during the recordings of the measurement signals.

After triggering the calculation by the synchronization signal, the FFT calculation for measurement signals was performed. The Hanning window was applied. Subsequently, the 4096 point FFT calculation for each signal was performed with the sampling frequency equal to $48 \mathrm{kS} / \mathrm{s}$ (frequency resolution: $11.7 \mathrm{~Hz}$ ). Such parameters provide sufficient spectral resolution for sound intensity calculations. The overlap was equal to $50 \%$. The FFT calculation was performed for each acoustic component $\left(p, u_{x}, u_{y}, u_{z}\right)$ separately. This operation yields transformed signals $X_{p}(i)$, $X_{u x}(i), X_{u y}(\mathrm{i}), X_{u z}(i)$ where $i$ (ranging from 0 to 4095) denotes the index of the spectral bin.

The matrix $\boldsymbol{X}$ (Eq. (3)) contains information about the arrival direction for every spectral component of the signal.

$$
\mathbf{X}=\left[\begin{array}{llll}
X_{p}(i) & X_{u x}(i) & X_{u y}(i) & X_{u y}(i)
\end{array}\right]
$$

or

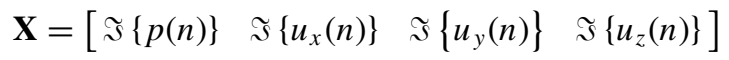

The sound intensity vector is defined and calculated according to Eq. (5).

$$
\vec{I}(i)=\left[\begin{array}{c}
I_{x}(i) \\
I_{y}(i) \\
I_{z}(i)
\end{array}\right]=\left[\begin{array}{l}
X_{p}(i) \cdot \overline{X_{u x}(i)} \\
X_{p}(i) \cdot \overline{X_{u y}(i)} \\
X_{p}(i) \cdot \overline{X_{u z}(i)}
\end{array}\right]
$$

where:

$I_{x}(i)$ - sound intensity component for $x$ direction for $i$-th spectral components,

$X_{p}(i)$ - coefficients of complex spectrum for $i$-th spectral components for acoustic pressure signal,

$\overline{X_{u x}(i)}$, conjugated spectrum coefficients for particle velocity in $x$ direction.

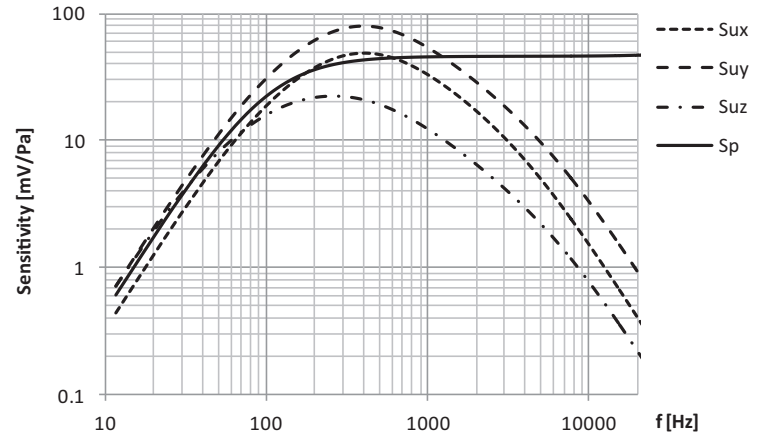

Fig. 6. Sensitivity characteristics of the pressure and particle velocity sensors.

Correct calculation of the sound intensity components required the sensitivity compensation of the AVS sensors in the full frequency range [22]. Equations (6) and (7) were applied to calculate the sensitivity of the particular sensors in relation to the frequency value. The sensitivity of the pressure sensor $\left(S_{P}\right)$ is given by:

$$
S_{p}(f)=S_{p, 1 k H z} \cdot \frac{\sqrt{1+\frac{f^{2}}{f_{c 3 p}^{2}}}}{\sqrt{1+\frac{f_{c l p}^{2}}{f^{2}}} \cdot \sqrt{1+\frac{f_{c 2 p}^{2}}{f^{2}}}}
$$

Particle velocity sensitivity $\left(S_{U}\right)$ is given by:

$$
S_{u}(f)=\frac{S_{u, 250 \mathrm{~Hz}}}{\sqrt{1+\frac{f_{c l u}^{2}}{f^{2}}} \cdot \sqrt{1+\frac{f^{2}}{f_{c 2 u}^{2}}} \cdot \sqrt{1+\frac{f^{2}}{f_{c 3 u}^{2}}} \cdot \sqrt{1+\frac{f_{c 4 u}^{2}}{f^{2}}}}
$$

As mentioned before, phase and sensitivity matched between the transducers over the frequency range of interest are critical for the accuracy of the results. The formula used for the correction phase between pressure and particle velocity is given by [22]:

$$
\begin{aligned}
\varphi_{p u}= & \arctan \left(\frac{C_{1 u}}{f}\right)-\arctan \left(\frac{f}{C_{2 u}}\right)-\arctan \left(\frac{f}{C_{3 u}}\right) \\
& +\arctan \left(\frac{C_{4 u}}{f}\right)-\arctan \left(\frac{C_{1 p}}{f}\right) \\
& -\arctan \left(\frac{C_{2 p}}{f}\right)-\arctan \left(\frac{f}{C_{3 p}}\right)
\end{aligned}
$$

Detailed values of the constants expressed in Eqs. (6-8) are specific for every probe (for the considered configuration, the values can be found in Calibration Report for the used probe [23]).

Based on the sensitivity equation and constant values, the correction characteristics were calculated. They are depicted in Fig. 6. For particle velocity sensors, the sensitivity unit was replaced from $[\mathrm{V} /(\mathrm{m} / \mathrm{s})]$ to its equivalent values expressed in $[\mathrm{mV} / \mathrm{Pa}]$.

All characteristics are strongly nonlinear, i.e., the amplitude and phase response are not flat, the response characteristics roll of high frequencies due to the thermal convention and inertia. To compensate for these nonlinearities, the correction functions were calculated. The equal broadband sensitivity $S_{c}$ was defined at $50[\mathrm{mV} / \mathrm{Pa}]$ for this purpose. 


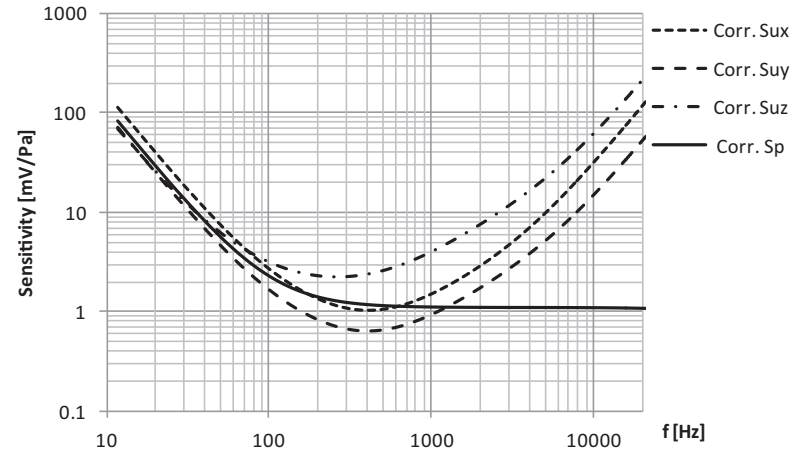

Fig. 7. Sensitivity correction characteristics of the pressure and particle velocity sensors.

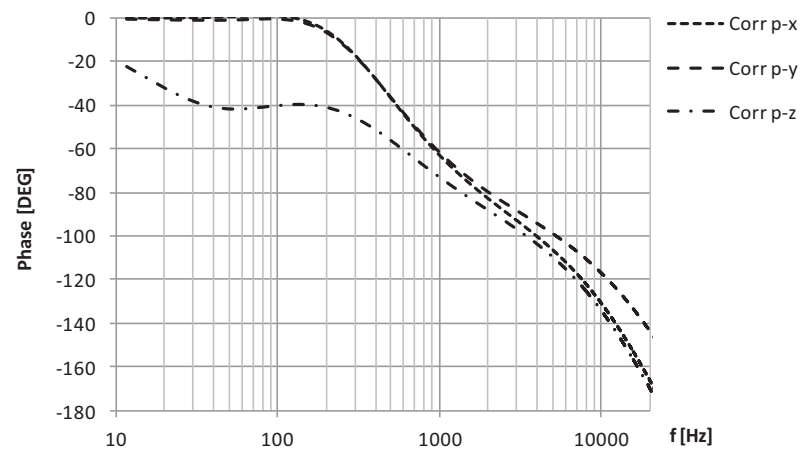

Fig. 8. Phase correction characteristics between pressure sensor and the given velocity sensor.

Correction characteristics (Corr. $\left.S_{x}\right)$ were calculated using the following formula:

$$
\operatorname{Corr} . S_{x}(f)=\frac{S_{c}}{S_{x}(f)}
$$

where: $S_{x}$ - sensitivity for the given sensor.

The final correction characteristics calculated using Eq. (9) is shown in Fig. 7. The function applied for the phase correction between the pressure sensor and the given velocity sensor calculated by means of Eq. (8) is shown in Fig. 8.

\section{EXPERIMENTAL RESULTS}

The performed measurements yielded sound intensity distribution characteristics (Fig. 9 - Fig. 11). They were grouped on the basis of the frequency value and the type of the loudspeaker configuration. Every figure series presents from the left: intensity distribution without the head, intensity distribution with the head, and the difference analysis that reveals the disturbance in the acoustic field induced by the head.

The white gaps present in all visualizations, correspond to the position of the artificial head.

In every graph, the intensity vectors obtained in the grid of measurement points are shown. The colored surface and the color scale corresponds to the magnitude of 3-D sound intensity vector expressed in $\mathrm{dB}$. The magnitude values between measurement points were calculated using linear interpolation (the interpolation process was performed before calculating the $\mathrm{dB}$ values).

The obtained visualizations show the simultaneous occurrence of a number of wave phenomena occurring in the vector acoustic flow field. The phenomena such as scattering, diffraction, and interference are well visible. They occurred for both low and high frequencies. What is very interesting is the fact that the acoustic shadow can be observed even for low frequencies, though causing very low attenuation (see Fig. 9 for details). Moreover, the wave phenomena occur not only close to the head, but its effects can be observed in some distance in front of and behind the head.

Using a single loudspeaker configuration measurement, the symmetry of the wave phenomena is well visible, but for two loudspeakers essential differences (in relation to the center loudspeaker) can be observed for frequencies 1000 and $4000 \mathrm{~Hz}$ (see Fig. 10 and Fig. 11 for details). For those frequencies, the wave phase begins to play the crucial role. When two loudspeakers were applied, the difference pattern of the field was completely different from the pattern observed for the center loudspeaker only (see Fig. 10(c), (d) and Fig. 11(c), (d) for details).

It is worth mentioning that we observed a real acoustic field reproduced by real loudspeakers. It means that the measured sound energy distribution depends on physical properties of applied sound sources. Its influence on the produced sound field can be responsible for an irregular energy pattern especially for higher frequencies (1000 and $4000 \mathrm{~Hz}$ ) observed in measurement results without and with the dummy head. However, the analysis resulting from "subtracting" two flow images, one without the head simulator and the other with the head, reduces the influence the speaker properties have on the observed wave phenomena.

\section{CONCLUSIONS AND FUTURE WORK}

This paper presented the system composed of a Cartesian robot, velocity transducers based on hot-wire anemometry, and the prepared software application for sound intensity measurements around the human head. Detailed results of sound intensity distribution for different loudspeaker configurations were included. The image of the acoustic field produced is the resultant effect of the obstacles appearing in a source radiation path, as well as the influence of scattered reflections together with their phase and amplitude relationship. Observation of acoustic wave distribution in the air and around a human head shows that phenomena occurring in the acoustic field are more complex than typically shown in acoustic field simulations. The wave phenomena such us diffraction and interference are clearly visible. Near the head simulator, we can observe local attenuation of sound energy in front of and behind the head, as well as the intensity level increase on both sides of the head. The latter phenomena are symmetrical and can be observed for all loudspeaker configurations. A particularly valuable 
(a)
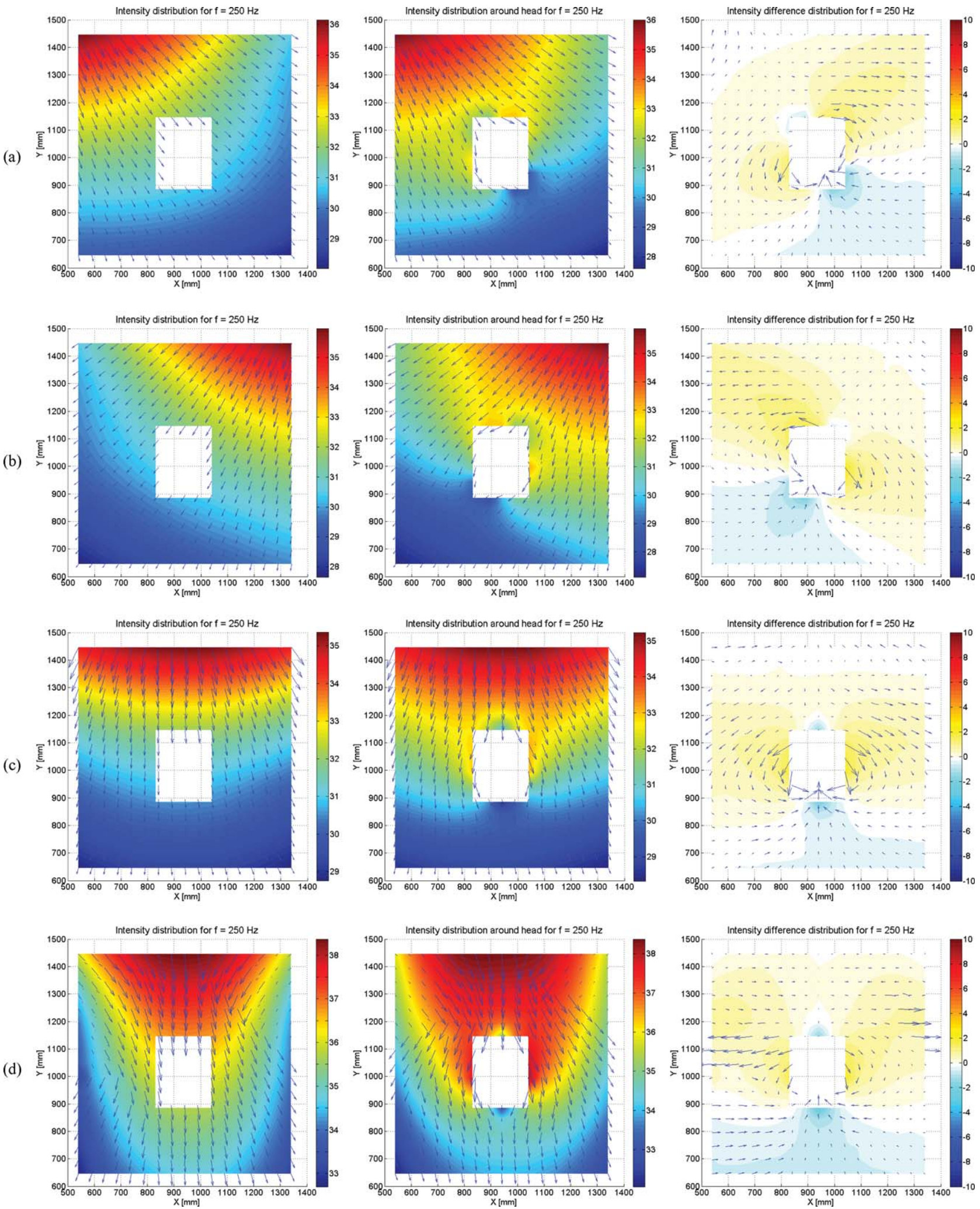

Fig. 9. Results for the: (a) first (signal radiated from the front left loudspeaker); (b) second (signal radiated from the front right loudspeaker); (c) third (signal radiated from the center loudspeaker); (d) fourth (signal radiated from the front left and right loudspeakers) loudspeaker configuration; frequency $250 \mathrm{~Hz}$. The wave phenomena such as diffraction and interference are clearly visible. Moreover, near the head, we can observe local attenuation of sound energy (in front of and behind the head), as well as increase of the intensity level on both sides of the head. These phenomena are symmetrical and can be observed for all loudspeaker configurations. 

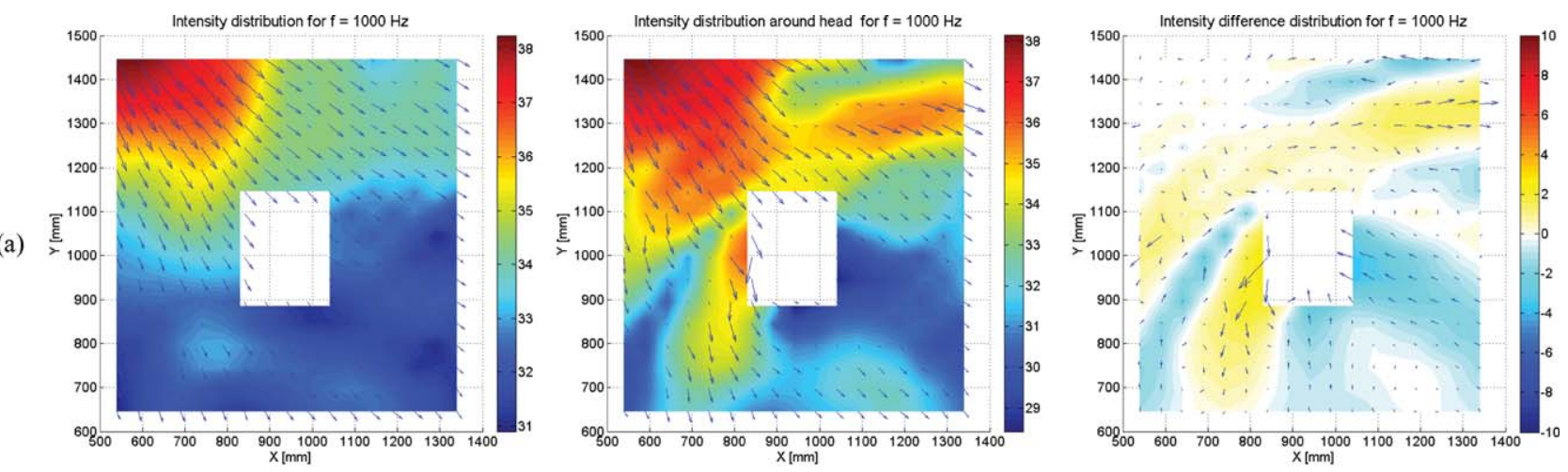

(b)
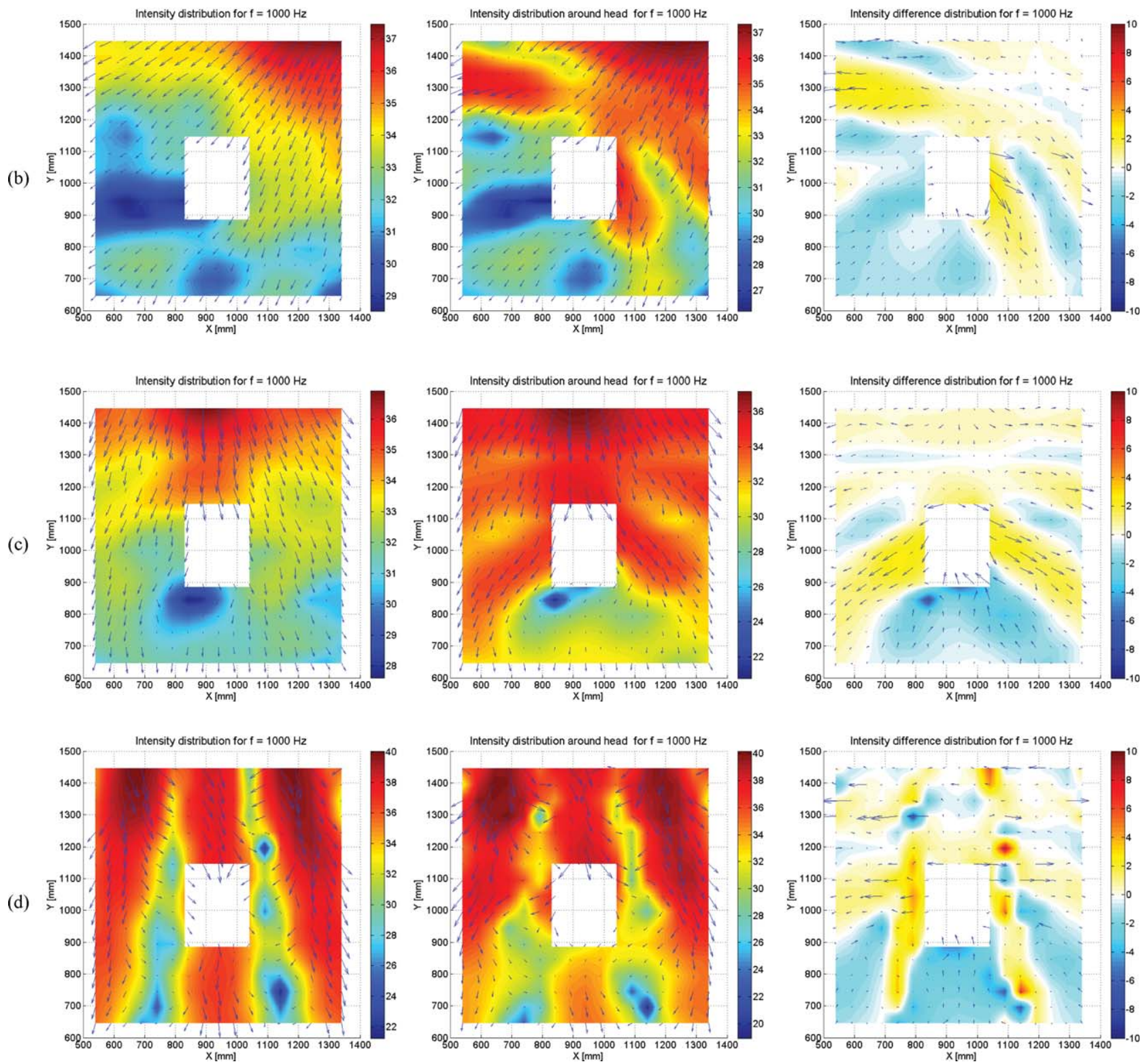

Fig. 10. Results for the: (a) first (signal radiated from the front left loudspeaker); (b) second (signal radiated from the front right loudspeaker); (c) third (signal radiated from the center loudspeaker); (d) fourth (signal radiated from the front left and right loudspeakers) loudspeaker configuration; frequency $1000 \mathrm{~Hz}$.

insight is provided by the illustration of the acoustic field in two loudspeaker configurations compared to the center sound image.

In future work sound intensity measurement results are to be compared with the sound field simulation techniques and mathematical modeling in order to achieve a satisfactory trade-off between mathematical accuracy and perceptual adequacy. Moreover, in the future research, the authors will try to visualize sound intensity distribution for common spatial sound reproduction techniques (5.1 system). The 
(a)

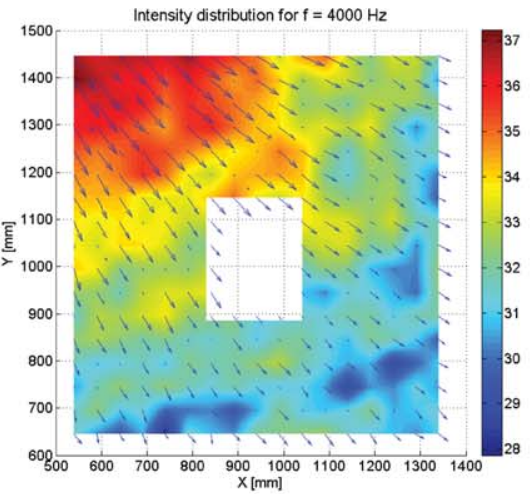

(b)

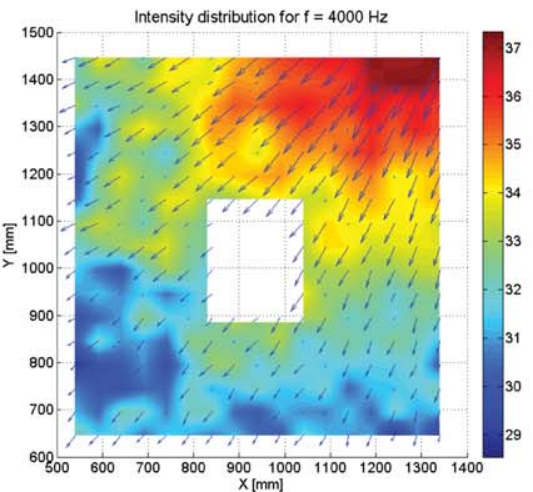

(c)

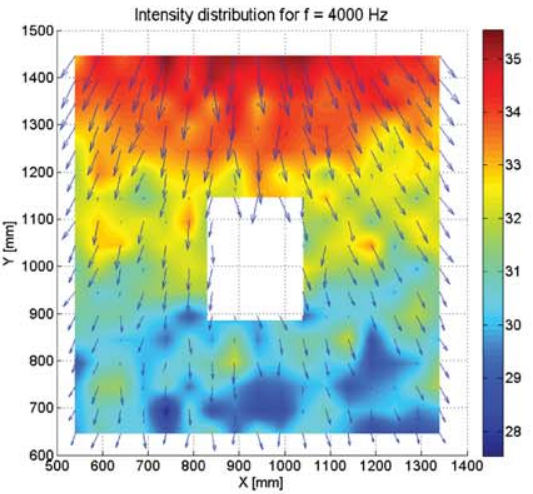

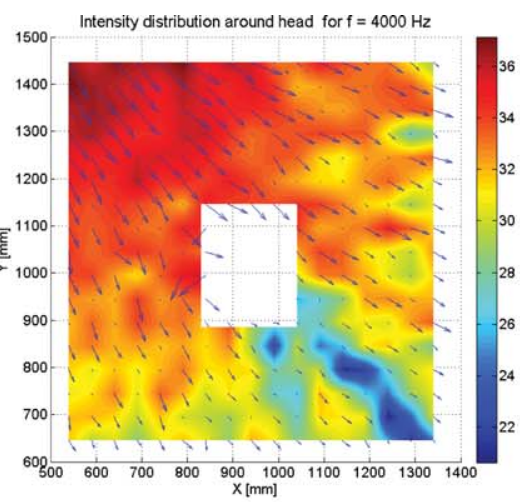
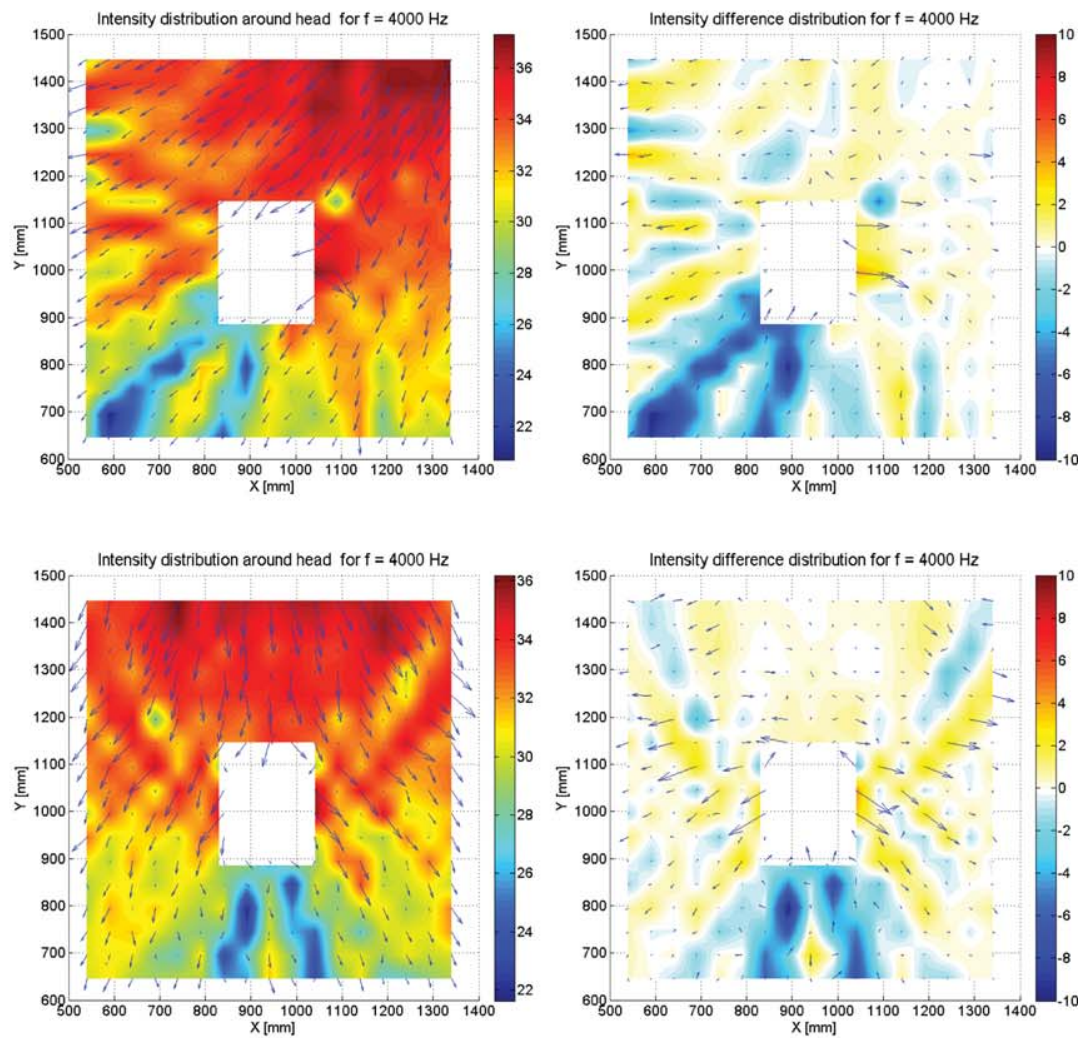

(d)
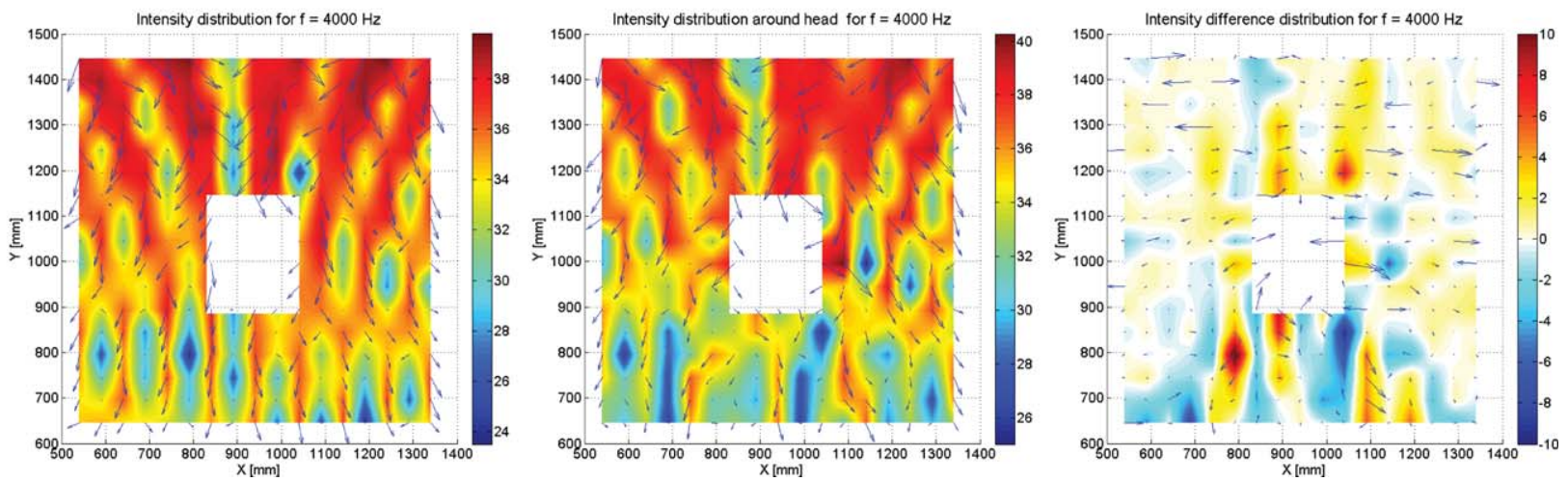

Fig. 11. Results for the: (a) first (signal radiated from the front left loudspeaker); (b) second (signal radiated from the front right loudspeaker); (c) third (signal radiated from the center loudspeaker); (d) fourth (signal radiated from the front left and right loudspeakers) loudspeaker configuration; frequency $4000 \mathrm{~Hz}$. 
amplitude panning and its influence on high frequencies will also be considered.

\section{ACKNOWLEDGMENTS}

The project was funded by the National Science Centre allocated on the basis of the decision DEC2012/05/B/ST7/02151.

\section{REFERENCES}

[1] "Multichannel Surround Sound Systems and Operations," AES Technical Document ESTD1001.0.01-05.

[2] H.-E. de Bree, "The Microflown: An Acoustic Particle Velocity Sensor,” Acoust. Aust., vol. 31, no. 3, pp. 91-94 (2003).

[3] R. Raangs, W. F. Druyvesteyn and H.-E. de Bree, "A Low Cost Intensity Probe" presented at the 110th Convention of the Audio Engineering Society (2001 May), convention paper 5292.

[4] W.-H. Cho, J.-G. Ih, and M. M. Boone, "Holographic Design of a Source Array Achieving a Desired Sound Field," J. Audio Eng. Soc., vol. 58, pp. 282-298 (2010 Apr.).

[5] S. Weyna, "Identification of Reflection and Scattering Effects in Real Acoustic Flow Field," Archives of Acoustics, vol. 28, no. 3, pp. 191-203 (2003).

[6] G. Cengarle and T. Mateos, "Comparison of Anemometric Probe and Tetrahedral Microphones for Sound Intensity Measurements," presented at the 130th Convention of the Audio Engineering Society (2011 May), convention paper 8363.

[7] W. Woszczyk, M. Iwaki, T. Sugimoto, K. Ono, and H.-E. de Bree, "Anechoic Measurements of ParticleVelocity Probes Compared to Pressure Gradient and Pressure Microphones," presented at the 122nd Convention of the Audio Engineering Society (2007 May), convention paper 7107.

[8] G. Cengarle, T. Mateos, and D. Bonsi, "A Second-Order Ambisonics Device Using Velocity Transducers," J. Audio Eng. Soc., vol. 59. pp. 656-668 (2011 Sep.).

[9] J. Merimaa, T. Lokki, T. Peltonen and M. Karjalainen, "Measurement, Analysis, and Visualization of Directional Room Responses," presented at the 111th Convention of the Audio Engineering Society (2001 Nov.), convention paper 5449.
[10] F. Jacobsen, "Sound Intensity and its Measurement and Applications," Acoustic Technology, Department of Electrical Engineering Technical University of Denmark (2011).

[11] F.J. Fahy Sound Intensity (Elsevier Applied Science, London, 1989).

[12] S. Weyna, "An Acoustics Intensity Based Investigation of the Energy Flow Over the Barriers," Acta Physica Polonica A. No. 1, Acoustic and Biomedical Engineering, vol. 118, pp. 172-178 (2010).

[13] J. Kotus, M. Plewa, and B. Kostek, "Measurements and Visualization of Sound Intensity Around the Human Head Using Acoustic Vector Sensor," presented at the 136th Convention of the Audio Engineering Society (2014 Apr.), e-Brief 154.

[14] Y. Kahana, "Numerical Modelling of the HeadRelated Transfer Function," Ph.D Thesis, University of Southampton, UK (2000).

[15] O. Kirkeby, P. A. Nelson and H. Hamada, "Local Sound Field Reproduction Using Two Closely Spaced Loudspeakers," J. Acoust. Soc. Am., vol. 104, no. 4, pp. 1973-1981 (1998).

[16] H. Wierstorf, A. Raake, M. Geier, and S. Spors, "Perception of Focused Sources in Wave Field Synthesis," J. Audio Eng. Soc., vol. 61, pp. 5-16 (2013 Jan./Feb.).

[17] A. Ando and K. Hamasaki, "Sound Intensity Based Three-Dimensional Panning" presented at the 126th Convention of the Audio Engineering Society (2009 May), convention paper 7675 .

[18] F. Jacobsen and H.-E. de Bree, "Measurement of Sound Intensity: p-u Probes versus p-p Probes," Proceedings of NOVEM (2005).

[19] F. Jacobsen and H.-E. de Bree, "A Comparison of Two Different Sound Intensity Measurement Principles," J. Acoust. Soc. Am., vol. 118, no. 3, pp. 1510-1517 (2005).

[20] J. Kotus, "Application of Passive Acoustic Radar to Automatic Localization, Tracking and Classification of Sound Sources," Information Technologies, vol. 18, pp. 111-116 (2010).

[21] J. Kotus, "Multiple Sound Sources Localization in Free Field Using Acoustic Vector Sensor,' Multimedia Tools and Applications, DOI 10.1007/s11042-013-1549-y, published online, 06, 2013.

[22] Microflown Technologies, Calibration Report of USP Probe, UT-0907-64, 17.07.2009.

[23] Microflown Technologies, Calibration Report of USP Probe, UR-900260-10, 25.06.2013. 


\section{THE AUTHORS}

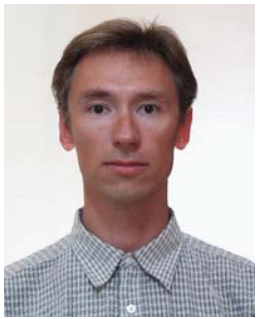

Józef Kotus

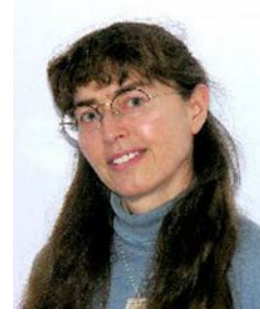

Bożena Kostek

Józef Kotus graduated from the Faculty of Electronics Telecommunications and Informatics, Gdansk University of Technology in 2001. In 2008 he completed his Ph.D. under the supervision of prof. Bożena Kostek. His Ph.D. work concerned issues connected with application of information technology to the noise monitoring and prevention of the noise-induced hearing loss. He is a member of the Audio Engineering Society (AES) and European Acoustics Association (EAA). He is an author and co-author of more than 70 scientific publications, including 11 articles from the ISI Master Journal List and 32 articles in the reviewed papers. Also four chapters co-authored by him were published by Springer. He has extensive experience in sound and image processing algorithms.

Bozena Kostek holds professorship at the Faculty of Electronics, Telecommunications and Informatics, Gdansk University of Technology (GUT), Poland. She is Head of the Audio Acoustics Laboratory. She received her M.Sc. degrees in sound engineering (1983) and organization and management (1986) from GUT. She also received post-

graduate DEA degree (1988) from Toulouse University, France. In 1992 she supported her Ph.D. thesis with honors at GUT, and in 2000 her D.Sc. degree at the Research Systems Institute, Polish Academy of Sciences. In 2005 the President of Poland granted her the title of Professor. She published over 500 scientific papers in journals and at international conferences. Under her guidance, $11 \mathrm{Ph} . \mathrm{D}$. students supported their doctoral theses and she supervised over 170 M.Sc. theses. She serves as the Editor-in-Chief of the Journal of the Audio Engineering Society since 2011. She was the recipient of many prestigious awards for research, including those of the Prime Minister of Poland for outstanding research achievements, prizes of the Polish Academy of Sciences and Ministry of Science, and the Bachelor Cross of the Polonia Restituta Order (2011). She received the Audio Engineering Society Fellowship Award in 2010 and the AES Citation in 2013. In 2013 Prof. Kostek was been elected as a member of the Polish Academy of Sciences. In 2003-2007 and 2009-2011, she was elected Vice-President of the Audio Engineering Society for Central Europe. In 2007-2009 and in 2011-2013 she served as the AES Governor. 